\title{
Hadronic Interactions at Cosmic Ray Energies
}

\author{
S. Ostapchenko ${ }^{a b}$

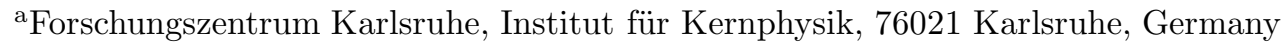 \\ ${ }^{\text {b}}$ D.V. Skobeltsyn Institute of Nuclear Physics, Moscow State University, 119992 Moscow, Russia
}

General physics of very high energy hadronic interactions is discussed. Special attention is payed to the contribution of semihard processes to the interaction dynamics and to the role of parton shadowing and parton density saturation. In particular, the implementation of non-linear interaction effects in the QGSJET-II model is discussed in detail. The predictions of the model are compared to selected accelerator data, including ones of the RHIC collider, and the relation to the calculated extensive air shower characteristics is discussed. Finally, the potential of accelerator and cosmic ray experiments for constraining model predictions is analyzed.

\section{INTRODUCTION}

During the last decade a significant progress has been achieved in experimental studies of high energy cosmic rays (CR) with extensive air shower (EAS) techniques, both concerning the measurements of the primary cosmic ray (PCR) energy spectrum and in determining the composition of cosmic radiation in the region of the spectral "knee" and at higher energies. This resulted from further development of the measurement and data analysis techniques, the latter including thorough EAS simulation studies with contemporary Monte Carlo (MC) tools. In EAS calculations a special role is played by hadronic MC generators which are used for the description of hadron-air and nucleus-air interactions in air showers, performing an extrapolation of current theoretical and experimental knowledge towards the highest $\mathrm{CR}$ energies. In particular, the QGSJET model [12, being based on the Gribov's effective Reggeon Field Theory (RFT) [3]4/5]6] and the Pomeron phenomenology 7/8, proved to be very successful in describing air shower data obtained by various experimental installations. The original versions of this MC generator have been developed as MC realizations of the Quark-Gluon String (QGS) model [7, including a generalization of the QGS model approach for the treatment of nucleus-nucleus collisions and a description of the fragmentation of nuclear spectator part 910. In QGSJET this has been supplemented by a phenomenological treatment of semihard processes, which result in the production of observed hadron jets of comparatively high transverse momenta. Finally, in the QGSJET-II model 11 a treatment of non-linear interaction effects has been developed, based on all-order re-summation of so-called enhanced (Pomeron-Pomeron interaction) RFT diagrams [12 13]. These approaches will be discussed below, in comparison with other models and with selected accelerator data.

\section{MODEL FRAMEWORK}

\subsection{Basic physics}

The general picture for high energy hadronic collision is the one of a multiple scattering process, being mediated by multiple parton (quark and gluon) cascades proceeding between the two hadrons. In the center of mass frame such parton cascades develop on a much larger time scale than the one of a parton-parton scattering, so that in the moment of the collision both projectile and target hadrons are represented by their parton clouds. Binary re-scatterings of some of these partons violate the coherence of the corresponding parton chains (consisting of the parton "parents" and "pre-parents"), which then fragment into secondary hadrons. Alternatively, the coherence may be preserved for some parton re- 


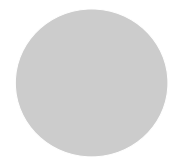

(a)

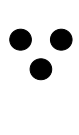

(b)

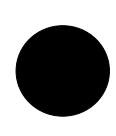

(c)

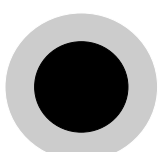

(d)
Figure 1. Proton profile as viewed in soft (a), hard (b), semihard (c), and general (d) interactions at very high energies.

scatterings, in which case all partons from the corresponding chains recombine back to their parent hadrons without a production of secondaries, which gives rise to elastic re-scattering processes. A general inelastic collision involves a number of elementary hadron production contributions as well as multiple elastic re-scatterings. In turn, elastic scattering is obtained when only elastic sub-processes occur.

With the energy increasing, the number of elementary re-scattering processes grows rapidly, due to the larger phase space for parton emissions. In addition, one expects a qualitative change in the structure of the underlying parton cascades. Indeed, at comparatively low energies all the partons are characterized by small transverse momenta; high $p_{t}$ emissions are suppressed by the smallness of the corresponding running coupling, $\alpha_{s}\left(p_{t}^{2}\right)$. By the uncertainty principle, each emission is characterized by a large displacement of the produced parton in the transverse plane, $\Delta b^{2} \sim 1 / p_{t}^{2}$. Thus, with the energy increasing further, such "soft" parton cascades rapidly expand towards larger impact parameters, while the density of partons per unit transverse area remains small, the hadron looking "grey", as depicted in Fig. 1 (a). However, at sufficiently high energies an important contribution comes from so-called "semi-hard" and "hard" parton cascades, in which some or all partons have comparatively high transverse momenta [14. There, the smallness of the strong coupling $\alpha_{s}\left(p_{t}^{2}\right)$ is compensated by a high parton density and by large logarithmic ratios of the longitudinal and transverse momenta for successive parton emissions. Purely hard cascades, which start, e.g., from valence quarks and contain only high $p_{t}$ partons, do not expand transversely,
$\Delta b^{2} \sim 1 / p_{t}^{2}$ being small, and lead to an increase of parton density in small areas ("hot spots") in the transverse plane - see Fig. 1 (b), while giving a negligible contribution to the total cross section. Contrary to that, typical semihard re-scatterings are two-step processes: first, parton branchings proceed with a small momentum transfer and the cascade develops towards larger impact parameters; next, high $p_{t}$ parton emissions become effective, leading to a rapid rise of the parton density at a given point in the transverse plane. As a result, the region of high parton density extends to large impact parameters (Fig. 1 (c)) and the contribution dominates in the very high energy limit. General hadronic interactions include all the mentioned mechanisms; hadrons in high energy collisions look as shown in Fig. 1(d): there is an extended "black" region of high density, dominated by the semihard processes, and around it there is a "grey" region of low density, formed by purely soft parton cascading [15. In the "black" region one expects strong non-linear parton effects to emerge, which result in the saturation of parton densities and in the suppression of soft parton emissions 14. On the other hand, such effects are negligible in the "dilute" peripheral region.

How to estimate the relative importance of the two regimes? Small peripheral contribution would correspond to the "black disc" limit for hadron-hadron scattering, with the ratio of elastic to total cross sections approaching $1 / 2$. The relative smallness of the observed $\sigma_{p p}^{\mathrm{el}} / \sigma_{p p}^{\text {tot }}$ ratio indicates that the "black" central region and the peripheral one are yet of comparable sizes [15.

In reality, the discussed separation of central and peripheral collisions is rather crude, as the average parton densities rise gradually with decreasing impact parameter. Thus, there exists an important "transition" region of moderately large impact parameters, characterized by large but not yet saturated parton densities, where the contributions of both soft and semihard processes are of equal importance, and where non-linear parton effects provide sizable corrections. In fact, it is this transition region which is expected to give the dominant contribution to the interaction characteristics relevant for EAS physics. 


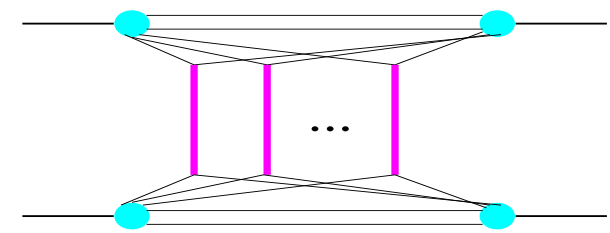

Figure 2. A general contribution to hadronhadron scattering amplitude. Elementary scattering processes (vertical thick lines) are described as Pomeron exchanges.

\subsection{Pomeron formalism}

As discussed above, general hadronic collisions necessarily involve emissions of soft low $p_{t}$ partons, which prevents one from applying the perturbative QCD formalism. However, for a number of key characteristics, like total and elastic cross sections or probabilities of various "macroscopic" configurations of inelastic interactions, the knowledge of the microscopic parton picture is not of extreme importance; the RFT allows one to calculate the quantities of interest based on the knowledge of the elastic amplitude for an "elementary" scattering process. For example, in the QGS model [7] hadron-hadron scattering is described as a multiple exchange of composite objects - Pomerons, as shown in Fig. 2. Each Pomeron represents a microscopic parton cascade whose precise description is not necessary at this stage. Rather one employs the theoreticallymotivated ansatz for the corresponding scattering amplitude:

$f_{a d}^{\mathrm{P}}(s, b)=\frac{i \gamma_{a} \gamma_{d} s^{\alpha_{\mathrm{P}}(0)-1} e^{-\frac{b^{2}}{4\left(R_{a}^{2}+R_{d}^{2}+\alpha_{\mathrm{P}}^{\prime}(0) \ln s\right)}}}{R_{a}^{2}+R_{d}^{2}+\alpha_{\mathrm{P}}^{\prime}(0) \ln s}$,

which is characterized by a power-like energy rise and by a logarithmically increasing slope; the parameters $\alpha_{\mathrm{P}}(0), \alpha_{\mathrm{P}}^{\prime}(0)$ are the intercept and the slope of the Pomeron Regge trajectory and $\gamma_{a}$, $R_{a}^{2}$ - the residue and the slope for the Pomeronhadron $a$ vertex 78 .

It is important to take into account the contributions of both elastic and inelastic intermediate hadron states between Pomeron emissions, such that the Pomeron-hadron vertex becomes a matrix corresponding to the transitions between those states. To diagonalize this matrix one con-

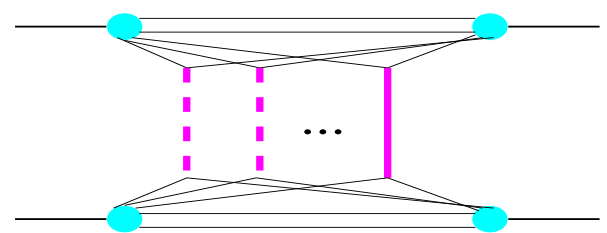

Figure 3. Typical inelastic interaction contains a number of elementary production processes, described by cut Pomerons (thick broken lines in the Figure), and any number of elastic re-scatterings - uncut Pomeron exchanges.

siders any hadron to be represented by a superposition of a number of elastic scattering eigenstates, $|a\rangle=\sum_{j} \sqrt{C_{a}^{(j)}}\left|j_{a}\right\rangle$, which are characterized by different absorption in the scattering process, $\gamma_{a}^{(j)}=\lambda_{a}^{(j)} \gamma_{a}$ [6]. Here $C_{a}^{(j)}$ and $\lambda_{a}^{(j)}$ are the weights of the eigenstates and the relative strengths for their coupling to the Pomeron; $\sum_{j} C_{a}^{(j)}=1, \sum_{j} C_{a}^{(j)} \lambda_{a}^{(j)}=1$. Then, the total cross section is obtained as the sum of partial scattering contribitions, being expressed via the so-called Pomeron eikonal $\chi_{a d}^{\mathrm{P}}(s, b)=\operatorname{Im} f_{a d}^{\mathrm{P}}(s, b)$

$\sigma_{a d}^{\mathrm{tot}}(s)=2 \int d^{2} b \sum_{j, k} C_{a}^{(j)} C_{d}^{(k)}\left(1-e^{-\lambda_{a}^{(j)} \lambda_{d}^{(k)} \chi_{a d}^{\mathrm{P}}(s, b)}\right)(2)$

To obtain cross sections for various final states, one makes use of the optical theorem, which relates the total sum of contributions of all final states to the imaginary part of the elastic amplitude for hadron-hadron scattering, hence, to the contributions of various unitarity cuts of elastic scattering diagrams. The so-called AbramovskiiGribov-Kancheli cutting rules [16] state that only certain classes of cut diagrams are important in the high energy limit and allow one to relate such contributions to particular final states of interest. For example, the cross sections for having $n$ simultaneous elementary production processes are described by the diagrams with $n$ "cut" Pomerons and any number of uncut ones, as shown in Fig. 3. whereas elastic and diffractive cross sections are obtained cutting the diagrams of Fig. 2 between the Pomerons, with no one being cut, and selecting elastic or diffractive intermediate hadron states in the cut plane. Thus, the cross sections 
for the $n$ cut Pomerons process and for the projectile diffraction dissociation read

$$
\begin{array}{r}
\sigma_{a d}^{(n)}(s)=\int d^{2} b \sum_{j, k} C_{a}^{(j)} C_{d}^{(k)} \\
\times \frac{\left[2 \lambda_{a}^{(j)} \lambda_{d}^{(k)} \chi_{a d}^{\mathrm{P}}(s, b)\right]^{n}}{n !} e^{-2 \lambda_{a}^{(j)} \lambda_{d}^{(k)} \chi_{a d}^{\mathrm{P}}(s, b)} \\
\sigma_{a d}^{\mathrm{DD}_{a}}(s)=\int d^{2} b \sum_{j, k, l, m}\left(C_{a}^{(j)} \delta_{j}^{l}-C_{a}^{(j)} C_{a}^{(l)}\right) \\
\times C_{d}^{(k)} C_{d}^{(m)} e^{-\left(\lambda_{a}^{(j)} \lambda_{d}^{(k)}+\lambda_{a}^{(l)} \lambda_{d}^{(m)}\right) \chi_{a d}^{\mathrm{P}}(s, b)}
\end{array}
$$

From (44) follows that a higher diffraction cross section is obtained for more asymmetric couplings $\lambda_{a(j)}$ of different eigenstates to the Pomeron. In turn, this results in a smaller total cross section and larger fluctuations in the number $n$ of elementary production processes (cut Pomerons), as one can see from (2) 3).

Having obtained partial probabilities of various inelastic final states, one has yet to describe particle production for each elementary inelastic process, which leads us back to the physics of the underlying parton cascade. However, one can employ here the string picture of the hadronization, based on the color structure of the corresponding final states. Namely, one assumes that each cut Pomeron process induces a color exchange between parton constituents of the interacting hadrons, such that color strings are stretched between them. With the two hadrons flying apart, these strings break up and hadronize, which is described by string fragmentation procedures. It is noteworthy, that in the QGS model the parameters for constituent parton momentum distributions and the ones for string fragmentation are not adjustable ones, being expressed via the characteristics of known Regge trajectories [ 7/17].

Remarkably, the described scheme can be generalized to hadron-nucleus and nucleus-nucleus interactions in a parameter-free way, both for cross section calculations and concerning the description of particle production [10. The only new input are nuclear density profiles, with the corresponding parameters being fixed by nuclear form factor measurements [18, individually for each nuclear type, as discussed in [19].

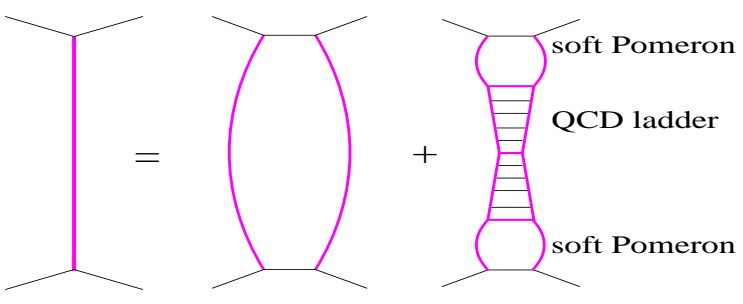

Figure 4. A general Pomeron is the sum of the "soft" and the "semihard" ones - correspondingly the 1st and the 2nd graphs in the r.h.s.

\subsection{Semihard processes}

As discussed in Section 2.1, at high enough energies a significant contribution to hadron-hadron scattering comes from semihard parton processes, where a part of the underlying parton cascade develops in the high $p_{t}$ region. To provide a microscopic treatment of the corresponding physics, one can apply the phenomenological Pomeron description to the low virtuality $\left(\left|q^{2}\right| \simeq p_{t}^{2}<Q_{0}^{2}\right)$ part of the parton cascade, while treating the high $p_{t}$ parton evolution using pQCD techniques, $Q_{0}^{2}$ being some chosen virtuality cutoff for QCD being applicable. Then, an elementary scattering process is described as an exchange of a "general Pomeron", which is a sum of the "soft" and the "semihard" ones, the latter being represented by a piece of QCD ladder sandwiched between two "soft" Pomerons [1220121, as shown in Fig. 4. As discussed in 2021, the upper and the lower half of the semihard Pomeron define parton (sea quark and gluon) momentum and impact parameter distributions in the projectile and target hadrons; the parameters for the coupling between the soft Pomeron and the ladder can be fixed by the data on hadron structure functions (SFs). The approach allows one to treat the semihard processes within the Pomeron scheme described in Section 2.2, the interaction cross sections are defined by the usual formulas, like (24), with the Pomeron eikonal being a sum of the soft and the semihard ones, $\chi_{a d}^{\mathrm{P}}(s, b)=\chi_{a d}^{\mathrm{P}_{\text {soft }}}(s, b)+\chi_{a d}^{\mathrm{P}_{\text {sh }}}(s, b)$. In turn, particle production procedure includes an explicit treatment of the high $p_{t}$ parton cascade, with the strings being formed between the produced final partons [2]20|21]. 


\subsection{Non-linear effects}

Developing a model for high energy hadronic interactions, one inevitably faces the problem of treating non-linear effects, connected to parton shadowing and saturation. Indeed, describing the interaction as a superposition of a number of re-scattering processes, mediated by parton cascades, one has to consider the case when such cascades overlap in the corresponding phase space and influence each other. Such effects are expected to be extremely important at very high energies and small impact parameters, i.e. in the "black" region of high parton densities, where they lead to the parton density saturation 14 and to significant reduction of secondary particle production. However, non-linear effects start to be efficient already at comparatively low energies and large impact parameters, the experimental indication being the rapid energy rise of the high mass diffraction cross section in the ISR energy range [22, the latter being just one of a number of manifestations of non-linear parton dynamics. Therefore, one has to develop a coherent description of the corresponding physics over a wide dynamic range, rather than restrict himself with a treatment of the saturation region.

Treating independent parton cascades effectively as Pomeron exchanges, the corresponding non-linear effects are described in the RFT as Pomeron-Pomeron interactions 23 24 25]. There, the main technical difficulty is to perform a resummation of contributions of the underlying enhanced Pomeron graphs, as more and more diagrams of compicated topologies come into play at higher energies. A re-summation method has been worked out recently [1213] and implemented in the QGSJET-II model [11]. The basic assumption of the approach was that PomeronPomeron coupling proceeds via parton processes at comparatively small virtualities $\left|q^{2}\right|<Q_{0}^{2}$ and can be described using phenomenological multiPomeron vertices of eikonal type [13. A reasonable consistency with relevant experimental data has been obtained using a fixed energyindependent $Q_{0}$-cutoff] 1 neglecting parton shad-

\footnotetext{
${ }^{1}$ The parameter set in 13 is different from the default QGSJET-II settings. While a general agreement with measured proton-proton cross section and proton SFs can
}

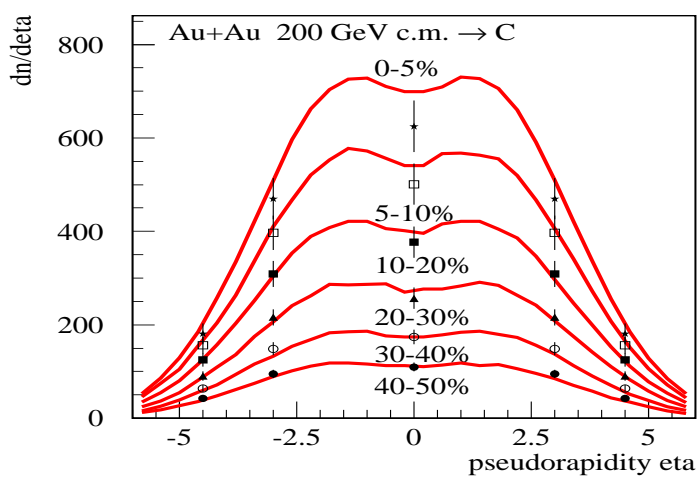

Figure 5. Pseudorapidity distributions of charged secondary particles in $\mathrm{Au}-\mathrm{Au}$ collisions of different "centralities"; QGSJET-II results (lines) are compared to BRAHMS data 27.

owing effects for $\left|q^{2}\right|>Q_{0}^{2}$. In particular, inferring the basic parameter of the scheme, the triplePomeron coupling, from HERA hard diffraction data, one obtains a satisfactory agreement with observed hadronic diffraction [13].

To describe particle production, one has to consider unitarity cuts of enhanced Pomeron diagrams and, for any configuration of cut Pomerons, to perform a full re-summation of uncut ones 13126. The big advantage of the developed procedure is that the solutions are obtained in the form of recursive equations, which can be implemented in a MC model and allow one to generate various configurations of interactions, including diffractive ones, in an iterative fashion [26].

The formalism seems to be adequate for the description of hadronic collisions in the peripheral and "transition" regimes. However, in the "black" region of high parton densities one may expect an important contribution of the "hard" $\left(\left|q^{2}\right|>Q_{0}^{2}\right)$ Pomeron-Pomeron coupling, which would provide additional screening corrections. A reasonable agreement of QGSJET-II with RHIC data on central heavy ion collisions (see Fig. 5) indicates the smallness of such effects. However, the situation may change at much higher energies.

be obtained for $Q_{0}^{2}$ as small as $1 \mathrm{GeV}^{2}$, the description of particle production requires a higher cutoff, the default QGSJET-II value being $2.5 \mathrm{GeV}^{2}$. This may be considered as an indication for significant parton shadowing in the virtuality range $1 \div 2.5 \mathrm{GeV}^{2}$. 


\subsection{Nuclear fragmentation}

Performing simulations of nucleus-induced air showers, one has to pay attention to the fragmentation of nuclear spectator part, as it has a significant influence on the predicted EAS fluctuations [10]. Nuclear fragmentation in QGSJET is strongly correlated with the interaction treatment; it is assumed that the excitation of the nuclear spectator part is proportional to the number of inelastically "wounded" nucleons. In case of comparatively low excitations, corresponding to peripheral interactions, the de-excitation proceeds via an evaporation of a certain number of nucleons or alpha-particles. Highly excited nuclei undergo multi-fragmentation, which is described as a percolation process: final fragments are formed by the clusters of nearby nucleons [10]. The scheme provides a reasonably good description of the mass yield dependence for all fragment masses, including intermediate ones, which is typically not the case if evaporation mechanism only is included (e.g., in [28]). Importantly, the relevant model parameters have been fixed with the data on nuclear fragmentation at energies above $2 \mathrm{AGeV}$, where the measured fragment yields are already stable and do not have any significant energy dependence. Correspondingly, the treatment can be safely extrapolated to cosmic ray energies.

\section{DISCUSSION OF MODEL RESULTS}

\subsection{Cross sections and inelasticity}

The longitudinal air shower development depends on a number of characteristics of hadronair interactions. While the largest effect comes from the inelastic proton-air (nucleus-air) cross section, which defines the position of the first interaction of the primary particle, pion-air and kaon-air cross sections and the inelasticities of hadronic interactions influence the shape of the characteristic EAS profile. In particular, cross sections of diffraction dissociation processes play here a special role. The diffraction of the target nucleus results in a negligible energy loss of the initial particle, secondary hadrons being produced in the target fragmentation region, and is essentially equivalent to a reduction of the inelastic cross section. The projectile hadron diffrac-
Table 1

Inelastic and non-diffractive hadron-carbon cross sections (in mb) at $200 \mathrm{GeV}$ lab. energy.

\begin{tabular}{lccc}
\hline & $\sigma_{n-12_{C}}^{\mathrm{inel}}$ & $\sigma_{p-12_{C}}^{\mathrm{ND}}$ & $\sigma_{\pi+-1{ }^{+} C}^{\mathrm{ND}}$ \\
\hline QGSJET-II & 238 & 224 & 167 \\
Experiment & $237 \pm 2[\underline{29}]$ & $225 \pm 7[\underline{30}$ & $171 \pm 5[\underline{30}$ \\
\hline
\end{tabular}

tion, being predominantly a coherent process, the target nucleus remaining intact with a high probability 10, results in a small energy loss of the leading particle, thus sizably influencing the overall inelasticity. It is noteworthy, that all the above-mentioned quantities mainly depend on the model description of hadronic collisions in the peripheral and "transition" regions. Indeed, at small impact parameters, hadrons already look "black", see Fig. 1 (d), with the interaction profile $\sigma_{h \text {-air }}^{\text {inel }}(s, b)$ (the probability of inelastic interaction at some impact parameter $b$ for a given energy $s$ ) being very close to unity, which still remains so when non-linear corrections to parton dynamics, e.g., parton saturation effects, are taken into account. This region does not contribute substantially to diffraction cross sections, as any rapidity gap, produced in some elementary production process, is covered by secondary particles created in other production sub-processes. Finally, the inelasticity can only weakly depend on the central region treatment. Central collisions involve a large number of production processes and lead in all cases to large energy losses of leading particles. Contrary to that, modifications of the treatment of the peripheral and the "transition" regions make a sizable effect on the predicted $\sigma_{h-\text { inel }}^{\text {ine }}(s, b)$ behavior, thus influencing the obtained values of $\sigma_{h \text {-air }}^{\text {inel }}$ and $\sigma_{h \text {-air }}^{\text {diffr }}$, and change the average number and fluctuations of the number of production sub-processes, which leads to large variations of the inelasticity.

As already mentioned, the generalization of the formalism, discussed in Sections 2.2 2.4, for hadron-nucleus and nucleus-nucleus interactions proceeds in a parameter-free way, which applies also to cross section calculations. For the latter, one obtains an excellent agreement with experimental data, some values given in Table 1 . The predictions of the SIBYLL 2.1 model [3132] 
for $\sigma_{h-\text { air }}^{\text {inel }}$ are close to the ones of QGSJET-II in the collider range but rise faster with energy. In principle, this may come from the fact that non-linear effects are introduced in SIBYLL for semihard processes only, being neglected for the "soft" component. As the latter plays the crucial role in peripheral collisions, which largely define the cross section behavior, one may expect that SIBYLL overestimates $\sigma_{h \text {-air }}^{\text {inel }}$ in the very high energy range. Nevertheless, model predictions depend also on the assumptions concerning hadron form factors (parton distributions in the transverse plane) and on the calibration to proton-proton data, as discussed in 33. Present uncertainties concerning the energy dependence of $\sigma_{h-\text { air }}^{\text {inel }}$ will be drastically reduced after the measurements of $\sigma_{p p}^{\text {tot }}$ at the LHC.

Model results for the inelasticity $K_{h \text {-air }}^{\text {inel }}$ hadron-air interactions are only constrained by fixed target accelerator measurements. Practically in all models the inelasticity rises with energy, due to the increase of the number of elementary production processes. However, the spread in the predicted $K_{h \text {-air }}^{\text {inel }}$ is rather large, around $20 \%$ in the high energy range. Additional constraints may come from the measurements of leading neutron spectra in proton-proton interactions by the LHCf experiment [34.

\subsection{Muon component of air showers}

An important topic are model predictions for the CR muon component. Here one has to distinguish between the results for inclusive muon spectra and for the muon content of air showers of a given primary energy. The former are dominated by single interactions of primary protons of energies in average only an order of magnitude higher than the ones of the measured muons. Due to the steepness of the primary CR spectrum, the corresponding results are very sensitive to the shape of the forward pion and kaon spectra in protonair collisions. The characteristic quantities are the so-called $Z$-factors [35, some values given in Table 2 for QGSJET-II and SIBYLL 2.1. Clearly, the two models predict different energy dependences for $Z_{\pi^{ \pm}}$: one observes a rather precise Feynman scaling in SIBYLL, which is supported by inclusive muon flux measurements [36],
Table 2

Pion and kaon $Z$-factors as predicted by the QGSJET-II and SIBYLL 2.1 models.

\begin{tabular}{lcccc}
\hline$E_{0}, \mathrm{TeV}$ & model & $Z_{\pi^{+}}$ & $Z_{\pi^{-}}$ & $Z_{K^{+}}$ \\
\hline 0.1 & QGSJET-II & 0.043 & 0.035 & 0.0036 \\
& SIBYLL 2.1 & 0.036 & 0.026 & 0.0134 \\
\hline \multirow{2}{*}{10} & QGSJET-II & 0.033 & 0.028 & 0.0034 \\
& SIBYLL 2.1 & 0.037 & 0.029 & 0.0097 \\
\hline
\end{tabular}

and a noticeable scaling violation in QGSJETII. Another difference is the higher values of $Z_{K^{+}}$ in case of SIBYLL, which is due to the harder kaon spectra in the model.

The EAS muon content is formed during a multi-step hadronic cascade process and mainly depends on the total multiplicity of hadron-air collisions, the shape of the forward pion spectra being of secondary importance. Model calibration is mainly performed at fixed target energies; in particular, both QGSJET-II and SIBYLL 2.1 appear to be consistent with recent data of the NA49 Collaboration [37. Concerning the predicted multiplicity, models agree with each other in the collider range; at the highest $\mathrm{CR}$ energies the characteristic differences reach a factor of three. Most likely, the situation will not improve significantly with the start of the LHC, as the spread in model results for the multiplicity of proton-proton interactions is yet at $10 \%$ level in the corresponding energy range.

However, one should not expect large differences between model predictions for the EAS muon number $N_{\mu}$. In particular, QGSJET-II and SIBYLL 2.1 results for $N_{\mu}$ differ by only $10 \div 15 \%$ in the high energy range. As shown in 38, changing the multiplicity of proton-air collisions by a factor of two, one obtains less than $10 \%$ modification of the predicted $N_{\mu}$. Of course, pion-air multiplicity is of greater importance here. However, in QGSJET-II one has little freedom to modify the latter. The only new parameters, defining the transition from proton-proton to pion-proton case, are the residue $\gamma_{\pi}$ and the slope $R_{\pi}^{2}$ of the Pomeron-pion coupling, which are reliably fixed by data on pion-proton total cross section and elastic scattering slope. The low $x$ behavior of the gluon and sea quark momentum distributions in pion are described by the same 
soft Pomeron asymptotics as in the proton case and the corresponding normalization is defined by the momentum sum rule, the valence quark distributions being fixed by measurements. It is therefore surprising that the new EPOS model 39] predicts significantly higher $N_{\mu}$, which is almost a factor of two in access of the QGSJET-II results at the highest CR energies [40]. Most likely, this is connected to the treatment of pion-air interactions in EPOS. The picture can be validated by the KASCADE experiment, where the muon component is measured directly, the corresponding analysis being sensitive to $10 \%$ variations in the predicted muon number [41].

The author acknowledges fruitful discussions with H.J. Drescher, R. Engel, and M. Strikman.

\section{REFERENCES}

1. N.N. Kalmykov, S.S. Ostapchenko and A.I. Pavlov, Bull. Russ. Acad. Sci. Phys. 58 (1994) 1966.

2. N.N. Kalmykov, S.S. Ostapchenko and A.I. Pavlov, Nucl. Phys. B (Proc. Suppl.) 52B (1997) 17.

3. V.N. Gribov, Sov. Phys. JETP 26 (1968) 414.

4. V.N. Gribov, Sov. Phys. JETP 29 (1969) 483.

5. M. Baker and K.A. Ter-Martirosian, Phys. Rep. 28 (1976) 1.

6. A.B. Kaidalov, Phys. Rep. 50 (1979) 157.

7. A.B. Kaidalov and K.A. Ter-Martirosyan, Sov. J. Nucl. Phys. 39 (1984) 979.

8. A. Capella et al., Phys. Rep. 236 (1994) 225.

9. N.N. Kalmykov and S.S. Ostapchenko, Sov. J. Nucl. Phys. 50 (1989) 315.

10. N.N. Kalmykov and S.S. Ostapchenko, Phys. Atom. Nucl. 56 (1993) 346.

11. S. Ostapchenko, Nucl. Phys. B (Proc. Suppl.) 151 (2006) 143.

12. S. Ostapchenko, Phys. Lett. B636 (2006) 40.

13. S. Ostapchenko, Phys. Rev. D74 (2006) 014026 .

14. L.V. Gribov, E.M. Levin and M.G. Ryskin, Phys. Rep. 100 (1983) 1.

15. M. Strikman, Nucl. Phys. Proc. Suppl., to be published; these proceedings.

16. V.A. Abramovskii, V.N. Gribov and O.V. Kancheli, Sov. J. Nucl. Phys. 18
(1974) 308.

17. A.B. Kaidalov, Sov. J. Nucl. Phys. 45 (1987) 902.

18. H. de Vries, C.W. de Jager and C. de Vries, Atomic Data and Nuclear Data Tables 36 (1987) 495.

19. N.N. Kalmykov, S.S. Ostapchenko and M.K. Alekseeva, Proc. of 26-th Int. Cosmic Ray Conf. (Salt Lake City), v. 1, p. 419, 1999.

20. H.J. Drescher et al., J. Phys. G25 (1999) L91.

21. S. Ostapchenko et al., J. Phys. G28 (2002) 2597.

22. K. Goulianos, Phys. Lett. B358 (1995) 379.

23. O.V. Kancheli, JETP Lett. 18 (1973) 274.

24. J.L. Cardi, Nucl. Phys. B75 (1974) 413.

25. A. Capella, J. Kaplan and J. Tran Thanh Van, Nucl. Phys. B105 (1976) 333.

26. S. Ostapchenko, in preparation.

27. I.G. Bearden et al. (BRAHMS Collab.), Phys. Rev. Lett. 88 (2002) 202301.

28. J. Engel et al., Phys. Rev. D46 (1992) 5013.

29. T.J. Roberts et al., Nucl. Phys. B159 (1979) 56.

30. A.S. Carroll et al., Phys. Lett. B80 (1979) 319.

31. R.S. Fletcher et al., Phys. Rev. D50 (1994) 5710 .

32. R. Engel et al., Proc. of 26-th Int. Cosmic Ray Conf. (Salt Lake City), v. 1, p. 415, 1999.

33. R. Engel, Nucl. Phys. B (Proc. Suppl.) 122 (2003) 40.

34. K. Kasahara, Nucl. Phys. Proc. Suppl., to be published; these proceedings.

35. T.K. Gaisser, Cosmic Rays and Particle Physics, Cambridge University Press, Cambridge, 1990.

36. M. Unger for the L3 Collab., Int. J. Mod. Phys. A20 (2005) 6928.

37. C. Alt et al. (NA49 Collab.), Eur. Phys. J. C45 (2006) 343.

38. S. Ostapchenko, Czech. J. Phys. 56 (2006) A149.

39. K. Werner, F.M. Liu and T. Pierog, Phys. Rev. C74 (2006) 044902.

40. K. Werner, F.M. Liu and T. Pierog, arXiv: astro-ph/0611311.

41. T. Antoni et al. (KASCADE Collab.), Astopart. Phys. 24 (2005) 1. 